\title{
Catalogando a palinoteca da Universidade Federal do Oeste do Pará como fonte de dados para estudos em palinologia
}

\author{
Cataloging the palynotheca of the Federal University of Western Pará as data source for studies in palynology
}

Raylana Rodrigues da Fonseca $1^{(1) \square}$, Jaílson Santos de Novais $2^{(0)}$, Chieno Suemitsu ${ }^{(6)}$ \& Vanessa Holanda Righetti de Abreu $4^{(1)}$

1. Programa de Pós-Graduação em Ciências Biológicas, Botânica Tropical, Universidade Federal Rural da Amazônia e Museu Paraense Emílio Goeldi, Belém, Pará, Brasil

\section{Programa de Pós-Graduação em Ciências e Tecnologias Ambientais, Instituto de Humanidades, Artes e Ciências Sosígenes Costa, Centro de Formação em Ciências Ambientais, Universidade Federal do Sul da Bahia, Porto Seguro, Bahia, Brasil \\ 3. Instituto de Ciências da Educação, Universidade Federal do Oeste do Pará, Santarém, Pará, Brasil}

4. Laboratório de Botânica e Palinologia, Instituto de Biodiversidade e Florestas, Universidade Federal do Oeste do Pará, Santarém, Pará, Brasil

\section{Palavras-chave}

Grãos de pólen. Melissopalinologia. Coleção biológica.

\section{Keywords}

Pollen grains. Melissopalynology. Biological collection.

\section{Doi}

doi.org/10.33447/paubrasilia.v3i2.47

Recebido em: 02/07/2020

Aceite em: 24/08/2020

Editor responsável: Gleidson V.

Marques (UFSB)

ISSN: 2595-6752

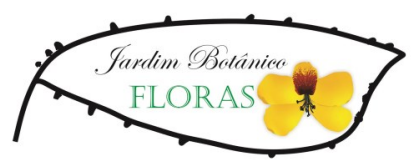

\section{Resumo}

Descrevemos a catalogação das lâminas palinológicas da palinoteca da Universidade Federal do Oeste do Pará. Atualmente, a palinoteca conta com 863 lâminas em seu acervo. Os trabalhos em palinologia aumentaram na universidade desde 2010, portanto, há mais de 1.000 lâminas para serem incorporadas à coleção. Essa coleção biológica tem subsidiado atividades de pesquisa e ensino de palinologia, enriquecendo o conhecimento da diversidade polínica regional, possibilitando o treinamento de estudantes de graduação e pós-graduação que exercem atividades na área. Todas as informações palinológicas estão sendo sistematizadas e serão disponibilizadas por meio de um site aberto, com a finalidade de promover a divulgação científica em palinologia. Esse é um importante avanço e contribui para desenvolver a palinologia na região amazônica.

\begin{abstract}
We describe the cataloging of the palynological slides at the palynotheca of the Federal University of Western Pará. Currently, the pollen slides collection has 863 slides. Works about palynology have been increasing in this university since 2010, therefore, there are over 1,000 slides to be incorporated into the collection. This biological collection has subsidized research activities and teaching palynology, enriching the knowledge about the regional pollen diversity, enabling the training of undergraduate and graduate students who work in the area. All palynological information is being systematized and will be available soon at an open access website, with the purpose of promoting the scientific dissemination of palynology. This is an important advance and contributes to developing the palynology in the Amazon region.
\end{abstract}

\section{Introdução}

A instrução normativa do IBAMA n. ${ }^{\circ} 160$, de 27 de abril de 2007, considera que uma coleção biológica reúne "material biológico testemunho (...) com o objetivo de gerar e subsidiar pesquisa científica ou tecnológica, bem como promover a cultura, a educação e a conservação do meio ambiente" (BRASIL, 2007, n.p.). De acordo com Marques e Lamas (2006) e Silva et al. (2017), quando bem organizadas, as coleções biológicas são valiosas fontes de informação e servem de base para o desenvolvimento de diversos tipos de estudos, como classificação (sistemática e taxonomia) e distribuição geográfica (biogeografia) dos organismos atuais e pretéritos, levantamentos faunísticos e florísticos, dentre outros estudos ecológicos. Gonzalez (2009) relata que estas informações ainda servem de base para orientar estudos sobre conservação de áreas nativas e recomposição de áreas degradadas, seja pela ação antrópica ou por perturbações naturais. 
A palinologia é um ramo da botânica que estuda as características morfológicas dos esporos, grãos de pólen e outras estruturas com parede orgânica resistente a ácidos. As palinotecas são coleções botânicas que conservam lâminas microscópicas com palinomorfos e podem ter perfis distintos, para contemplar as subáreas da palinologia e as necessidades de diferentes usuários(as), especialmente estudantes e pesquisadoras(es). Dentre as aplicações da palinologia que fazem uso das palinotecas, estão a palinotaxonomia, que corresponde ao uso dos grãos de pólen para caracterizar floras e subsidiar estudos taxonômicos e derivados; a melissopalinologia, que identifica a flora utilizada por abelhas melíferas e contribui com a caracterização microscópica de mel e outros produtos apícolas, por meio dos grãos de pólen; a palinoecologia, que estuda a dispersão polínica por meio abiótico ou biótico, especialmente pelos animais, naturalmente, um processo fundamental para a reprodução das plantas; e a paleopalinologia, que estuda grãos de pólen, esporos, acritarcas e quitinozoários fósseis encontrados em resíduos insolúveis resultantes de tratamentos físicos e químicos em rochas sedimentares (Fonseca; Vieira, 1984; Correia, et al., 2017).

$\mathrm{Na}$ região Norte do Brasil, grandes grupos de pesquisa desenvolvem trabalhos de forma sistemática e continuada na área da palinologia, como aqueles situados no Amazonas, no Instituto Nacional de Pesquisas da Amazônia (INPA), e no Pará, no Museu Paraense Emílio Goeldi (MPEG), na Embrapa Amazônia Oriental, na Universidade do Estado do Pará (UEPA) e na Universidade Federal do Pará (UFPA). Outros grupos localizam-se no Acre, na Universidade Federal do Acre (UFAC), e em Roraima, no Museu Integrado de Roraima (MIRR). O grupo mais recente na região está na Universidade Federal do Oeste do Pará (UFOPA), em Santarém (PA), cujas atividades iniciaram-se em 2010 (Novais et al., 2018).

Atualmente, o Laboratório de Botânica e Palinologia (LABOP) da UFOPA possui parcerias firmadas tanto com o INPA, quanto com o MPEG, a partir da coorientação de estudantes de pósgraduação que desenvolvem parte de seus trabalhos na UFOPA e parte nessas instituições parceiras. Além disso, a coleção palinológica da UFOPA mantém diálogo com o Núcleo de Especialistas em Palinologia da Sociedade Botânica do Brasil (Nepal/SBB), participando das reuniões promovidas pelo grupo durante os Congressos Nacionais de Botânica e dos levantamentos de coleções conduzidos por esse Núcleo da SBB. Esse reconhecimento é importante, pois permite o diálogo com outras palinotecas do país e parcerias com pesquisadoras(es) associadas(os) a tais coleções. Um exemplo disso são as constantes pesquisas desenvolvidas em colaboração entre o LABOP e a palinoFLORAS, a palinoteca do Jardim Botânico FLORAS da Universidade Federal do Sul da Bahia (UFSB).

$\mathrm{O}$ acervo de uma palinoteca precisa estar ativo e bem organizado, com os dados devidamente catalogados e as lâminas bem conservadas para serem consultadas por usuárias(os) internas(os) e visitantes. Além disso, constantemente novas lâminas precisam ser incorporadas à coleção, principalmente por conta do avanço das pesquisas nas diferentes áreas da palinologia, sendo fundamental, garantir um plano de manutenção e controle do acervo. Diante disso, consideramos necessário manter, formalizar e divulgar a coleção biológica destinada à palinologia na UFOPA, bem como disponibilizar todas as informações pertinentes ao material depositado na coleção ao público interessado. Sendo assim, a comunidade científica e demais profissio- nais de áreas aplicadas poderão realizar diversos tipos de estudos afins à palinologia, com suporte da palinoteca da UFOPA.

Portanto, o presente trabalho objetivou catalogar o laminário e apresentar a organização da palinoteca do Laboratório de Botânica e Palinologia da UFOPA. Além disso, buscou descrever o estágio atual e o processo de incorporação de novas lâminas à coleção, a fim de difundir os dados palinológicos estudados pelo corpo docente e discente da Universidade. Dessa forma, permitirá sistematizar os dados científicos produzidos a partir da coleção palinológica do Baixo Amazonas para que, futuramente, haja manutenção adequada desse acervo.

\section{Palinoteca da UFOPA}

\section{O inicio das atividades}

A palinoteca da UFOPA foi implementada em 2010, logo após a criação da Universidade, sob a curadoria do professor Jaílson Novais, com a colaboração de estudantes de iniciação científica e da professora Chieno Suemitsu. À época, a professora Suemitsu coordenava o Laboratório de Botânica Taxonômica (LABOT) e era curadora do Herbário de Santarém (HSTM), ambos vinculados ao atual Instituto de Ciências da Educação da UFOPA. Durante alguns anos, a palinoteca permaneceu vinculada ao LABOT e vários estudos foram nela desenvolvidos, ainda que com pouca infraestrutura. O processamento laboratorial e algumas análises microscópicas das amostras eram feitos com apoio do Laboratório de Química, do Laboratório de Ensino de Biologia e do Laboratório de Tecnologia da Madeira da UFOPA. Muitas amostras também foram processadas em laboratórios de instituições parceiras, como o Instituto Nacional de Pesquisas da Amazônia, no Amazonas, e a Universidade Estadual de Feira de Santana, na Bahia.

Em 2014, ingressou na universidade a professora Vanessa $\mathrm{H}$ $\mathrm{R}$ de Abreu, e iniciou a parceria de pesquisa com o professor Novais. A partir de 2015, com a redistribuição do professor Novais para a UFSB, foi criado o Laboratório de Botânica e Palinologia (LABOP) da UFOPA, vinculado ao Instituto de Biodiversidade e Florestas (IBEF), onde toda a coleção da palinoteca ficou instalada e, desde então, a mesma ficou sob a curadoria da professora Abreu, onde permanece até os dias atuais.

\section{O laboratório}

O Laboratório de Botânica e Palinologia está vinculado ao Instituto de Biodiversidade e Florestas e situa-se no Núcleo Tecnológico de Bioativos, na Unidade Tapajós da UFOPA, em Santarém (PA). No presente laboratório encontra-se a palinoteca, contendo lâminas de microscopia acondicionadas em caixas de polipropileno ou madeira, específicas para acondicionar esse tipo de amostra. As lâminas estão separadas por data de coleta do material nelas depositado. Atualmente, o LABOP possui alguns equipamentos que são rotineiramente usados em estudos palinológicos, como balanças analíticas de precisão, banhos-maria digitais, câmera digital, centrífuga, chapas aquecedoras, estereomicroscópios, estufa, geladeira, microscópios ópticos com e sem câmera acoplada. 
Laminário

Para preparar as lâminas das análises melissopalinológicas, o LABOP adota como recomendação prévia à acetólise o tratamento descrito por Jones e Bryant (2004) e modificações de Novais et al. (2013), utilizando etanol a 95\% (ETOH) para a diluição inicial do mel, como um esforço para reduzir a perda de grãos de pólen durante a eliminação do líquido sobrenadante. E, posteriormente, segue-se o método de acetólise de Erdtman (1952).

Nos estudos de palinotaxonomia, o LABOP adota o método de acetólise de Erdtman (1952), técnica aplicada para preparar os grãos de pólen de forma que a sua parede (exina) seja melhor visualizada, pois ocorrerá a retirada da intina (conteúdo citoplasmático). Isso ocorre por meio de técnica que consiste em uma série de etapas químicas, utilizando-se os seguintes produtos: ácido acético glacial, anidrido acético, ácido sulfúrico, acetona, água destilada e água glicerinada a 50\%.

As lâminas depositadas na coleção são permanentes e os grãos de pólen acetolisados são montados em gelatina glicerinada de Kisser (1935 apud Erdtman, 1952). Em geral, são preparadas ao menos três lâminas por amostra, de modo a uniformizar a amostra (Salgado-Labouriau et al., 1965). Atualmente, o acervo da palinoteca do LABOP conta com 863 lâminas catalogadas. E, como os trabalhos em palinologia são intensos na universidade, há mais de 1.000 lâminas para serem incorporadas à coleção.

Etiquetagem e catalogação

As lâminas provenientes dos estudos palinológicos são etiquetadas de forma a uniformizar a coleção biológica e são separadas por famílias botânicas (palinotaxonomia), espécies de abelhas (melissopalinologia) e interações ecológicas (palinoecologia). Posteriormente, as lâminas são contadas e acondicionadas em caixas porta-lâminas. As lâminas são visualizadas em microscopia de luz e os grãos de pólen e tipos polínicos são caracterizados e identificados, sempre que possível, até a categoria taxonômica de espécie.

Atualmente, todo o material do laminário está catalogado em tabelas no software Microsoft Excel $2010^{\circledR}$, para facilitar a consulta ao acervo da palinoteca. As fotomicrografias e todas as informações palinológicas obtidas estão sendo disponibilizadas em um site (https://labopufopa.wixsite.com/ibefufopa) desenvolvido em parceria com o projeto "Informatização e elaboração de website da palinoteca da Universidade Federal do Oeste do Pará - UFOPA”. No site, já podemos encontrar informações sobre o laboratório e identificações palinológicas. Embora ativo, o site está em processo de atualização e será alimentado constantemente.

\section{Descrição palinológica}

A descrição morfológica dos tipos polínicos encontrados nas lâminas dos estudos do LABOP geralmente seguem o padrão e a terminologia usuais em palinologia, usando como fontes básicas Erdtman (1952), Salgado-Labouriau (1973), Barth e Melhem (1988), Melhem et al. (2003), Punt et al. (2007) e Hesse et al. (2009). No banco de dados constam informações como o nome da família, gênero e/ou espécie do tipo polínico e a espécie das abelhas.
Para a caracterização taxonômica dos grãos de pólen, especialmente em estudos aplicados, como os melissopalinológicos, é adaptado o conceito de "tipo polínico" proposto por Joosten e De Klerk (2002) e De Klerk e Joosten (2007). As medições e fotomicrografias dos tipos polínicos são obtidos em microscópio Zeiss PrimoStar e por meio do programa de captura de imagens AxionCam ICc.

\section{Dados atuais da palinoteca}

O laminário da palinoteca do LABOP/UFOPA, até o início deste estudo, registrava em seu acervo um total de 863 lâminas de microscopia, etiquetadas e acondicionadas em caixas porta-lâminas de plástico e/ou madeira (Figura 1). Havia cerca de 1.112 lâminas ainda não depositadas na coleção. Desse total, 841 lâminas foram incorporadas à palinoteca ao longo do presente trabalho, registradas em um banco de dados compartilhado no Microsoft Access $2010^{\circledR}$ para facilitar a consulta ao acervo. As 271 lâminas restantes pertencem a pesquisas palinológicas em andamento.

Todas as lâminas, depositadas e a depositar na coleção, totalizam 1.975 registros. Com o intuito de controlar a quantidade de lâminas pertencentes aos distintos perfis da palinoteca, foi realizada a contagem e divisão do laminário por áreas de estudos desenvolvidos na UFOPA, desde 2010. Dos trabalhos realizados em melissopalinologia, foram contabilizadas 1.286 lâminas; em palinotaxonomia, somaram-se 246 lâminas; e em palinoecologia, 172 lâminas (Figura 2). As lâminas de trabalhos em andamento não estão aqui incluídas, pois as análises estão em processamento, não sendo possível adicionar as lâminas à coleção no momento.

Figura 1. Organização da palinoteca no Laboratório de Botânica e Palinologia da UFOPA: a. Acondicionamento dos laminários em arquivo de aço; b, c, d, e. Lâminas de microscopia etiquetadas e acondicionadas em laminários físicos, de forma a uniformizar a coleção biológica.
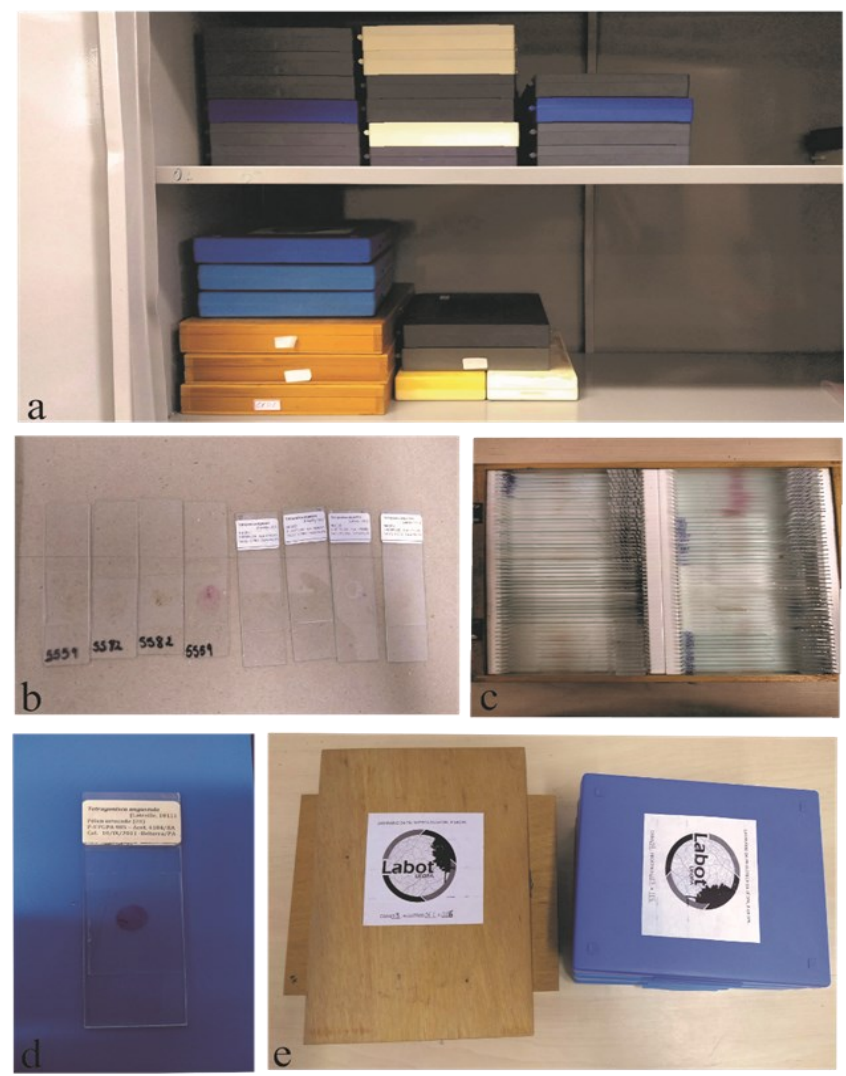
Figura 2. Quantidade de lâminas da palinoteca da UFOPA, dividida por área de estudo em palinologia.

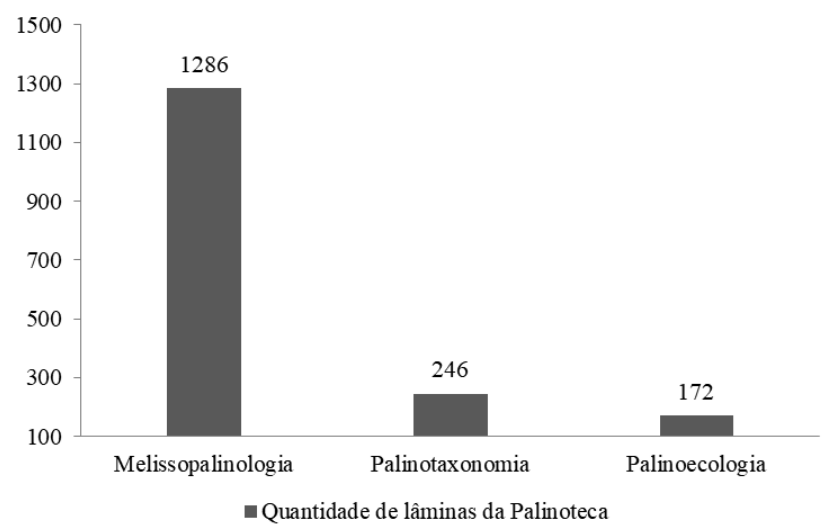

Os trabalhos realizados pelo LABOP/UFOPA compreendem estudos de grãos de pólen encontrados no mel de abelhas e nos produtos derivados, como própolis, pólen estocado e pólen corbicular. As espécies de abelhas trabalhadas no laboratório até o momento são: Centris (Centris) aenea Lepeletier, 1841; Centris (Melacentris) atriventris Mocsáry, 1896; Centris (Ptilotopus) denudans Lepeletier, 1841; Centris (Centris) flavifrons Fabricius, 1775; Frieseomelitta longipes Smith, 1854; Melipona (Melikerria) interrupta Latreille, 1811; Melipona (Michmelia) seminigra aff. pernigra Moure e Kerr, 1950; Scaptotrigona aff. xanthotricha Moure, 1950; Tetragonisca angustula Latreille, 1811; Xylocopa (Neoxylocopa) carbonaria Smith, 1854 e Xylocopa (Neoxylocopa) frontalis Olivier, 1789.

As famílias botânicas mais representadas da palinoteca atualmente são: Amaranthaceae, Anacardiaceae, Apocynaceae, Araliaceae, Arecaceae, Asteraceae, Combretaceae, Cyperaceae, Euphorbiaceae, Fabaceae, Loranthaceae, Malpighiaceae, Malvaceae, Melastomataceae, Meliaceae, Myrtaceae, Rubiaceae, Salicaceae e Sapindaceae. O gênero mais representado na coleção é Mimosa L., da família Fabaceae, representando a família com maior número de tipos polínicos na coleção. Isso é o esperado, pois, de acordo com Marques-Souza et al. (2002), a família Fabaceae possui espécies abundantes e com ampla distribuição em toda a Amazônia. São espécies nativas que têm grande influência na alimentação das abelhas sem ferrão, polinizadores de extrema importância para a flora local.

Para favorecer o livre acesso às informações sobre a descrição palinológica das espécies vegetais representadas na palinoteca, estão sendo elaborados palinogramas no software Corel DRAWX $7^{\circledR}$, que serão disponibilizados na palinoteca virtual (Figura 3).

As espécies vegetais atualmente representadas no acervo são aqui listadas de acordo com as categorias da Lista Vermelha de Espécies Ameaçadas de Extinção, da União Internacional para a Conservação da Natureza (IUCN, 2020), com o intuito de informar sobre o status de conservação das espécies de plantas encontradas na região amazônica e que estão presentes no acervo da palinoteca da UFOPA (Tabela 1).

A classificação usada para as espécies, de acordo com a IUCN (2020), foi: dados insuficientes (DD) - um táxon listado nesta categoria ocorre quando as informações são insuficientes para que se faça uma avaliação direta ou indiretamente de seu risco de extinção, portanto, são necessárias mais informações e isso não exclui a possibilidade de que pesquisas futuras demonstrem que este táxon poderá ser classificado em uma categoria de ameaça; pouco preocupante (LC) -quando foi avaliado de acordo com os critérios e não se qualificou para as demais categorias de risco; são incluídas nesta categoria os táxons abundantes e de ampla distribuição e vulnerável (VU) -quando corre alto risco de extinção na natureza (Tabela 1).

No acervo da palinoteca do LABOP/UFOPA estão representadas: uma espécie para a categoria "dados insuficientes" (Mangifera indica L. - Anacardiaceae), 31 espécies na categoria "pouco preocupante" e uma espécie na categoria "vulnerável" (Swietenia macrophylla King. - Meliaceae) (Tabela 1). Esta última é conhecida pelos nomes comuns de mogno-brasileiro ou aguano e está classificada na categoria vulnerável por ser uma espécie de alto valor econômico, que sofreu grande exploração e, conforme Costa (2013), os estudos para essa espécie são dirigidos em busca de alternativas para cultivo e manejo, principalmente em sistemas agroflorestais.

Atualmente, o corpo discente da palinoteca do LABOP/ UFOPA é composto por nove estudantes, sendo seis de graduação (1 IC e 5 TCC) e três vinculados a programas de pós-graduação - 2 mestrados (MPEG e INPA) e 1 doutorado (INPA) - (Tabela 2). Muitos desses trabalhos são apresentados em eventos científicos, com o intuito de divulgar as produções da instituição e despertar o interesse de novas(os) alunas(os) para a pesquisa em palinologia.

A iniciativa de estruturar e catalogar o laminário da palinoteca acompanha trabalhos similares no país, como em Novais et al. (2018), Gonçalves-Esteves et al. (2014) e Luz et al. (2014). A posterior disponibilização desses dados em ambiente virtual permitirá a consulta detalhada ao acervo, desde lâminas etiquetadas e referenciadas até o acesso às imagens de grãos de pólen e esporos.

As pesquisas em melissopalinologia destacam-se na UFOPA, dentre os demais ramos da palinologia. Esses estudos vêm contribuindo para entender a relação abelhas-flores na região amazônica e os resultados também são aplicados em atividades de educação ambiental e na meliponicultura local, atividade que, segundo VillasBoas (2012), vem crescendo na Região Norte do Brasil.

Os estudos palinológicos incentivam a atividade da meliponicultura sustentável na região, especialmente os estudos realizados na Reserva Extrativista Tapajós-Arapiuns e no Projeto de Assentamento Agroextrativista do Lago Grande, no município de Santarém. Mediante isso, compreendemos a importância de gerar conhecimentos detalhados acerca das espécies botânicas representadas no acervo, permitindo, assim, subsidiar ações de conservação e uso sustentável da biodiversidade, sendo este nosso objetivo de trabalho.

A palinoteca do LABOP/UFOPA trabalha para ampliar e informatizar seu acervo, tendo iniciado recentemente o processo de digitalização dos dados da coleção, a fim de disponibilizá-los para consulta on-line, visando divulgar as pesquisas na área, como um retorno para a comunidade em geral. Segundo Gonçalves-Esteves et al. (2014), as palinotecas do Brasil vêm se reinventando ao buscar novas formas de ampliar os acervos e, no que se refere à informatização, buscam investir em material permanente com alta tecnologia e organização avançada. Tudo isso para desenvolver pesquisas e formar pessoal especializado em diferentes áreas da palinologia. De acordo com Oliveira e Lorscheitter (2014), a construção de um banco de dados fornecendo fotomicrografias com escalas, nome científico de cada espécie vegetal e informações morfológicas dos palinomorfos constitui um material de referência indispensável para trabalhos relacionados à palinologia em qualquer região. 
Figura 3. Exemplo de palinograma elaborado nos estudos em palinotaxonomia (a-d), palinoecologia (e-f) e melissopalinologia (g): a. Anacardiaceae - Spondias mombin L.; b. Burseraceae - Protium heptaphyllum (Aubl) Marchand; c. Myrtaceae - Eugenia brasiliensis Lam.; d. Sapindaceae - Serjania tipo; e. Loranthaceae - Psittacanthus encalyptifolius; f. Passifloraceae - Passiflora tipo; g. Melastomataceae - Miconia tipo (círculo) e Sapindaceae - Talisia esculenta (ponta de seta).
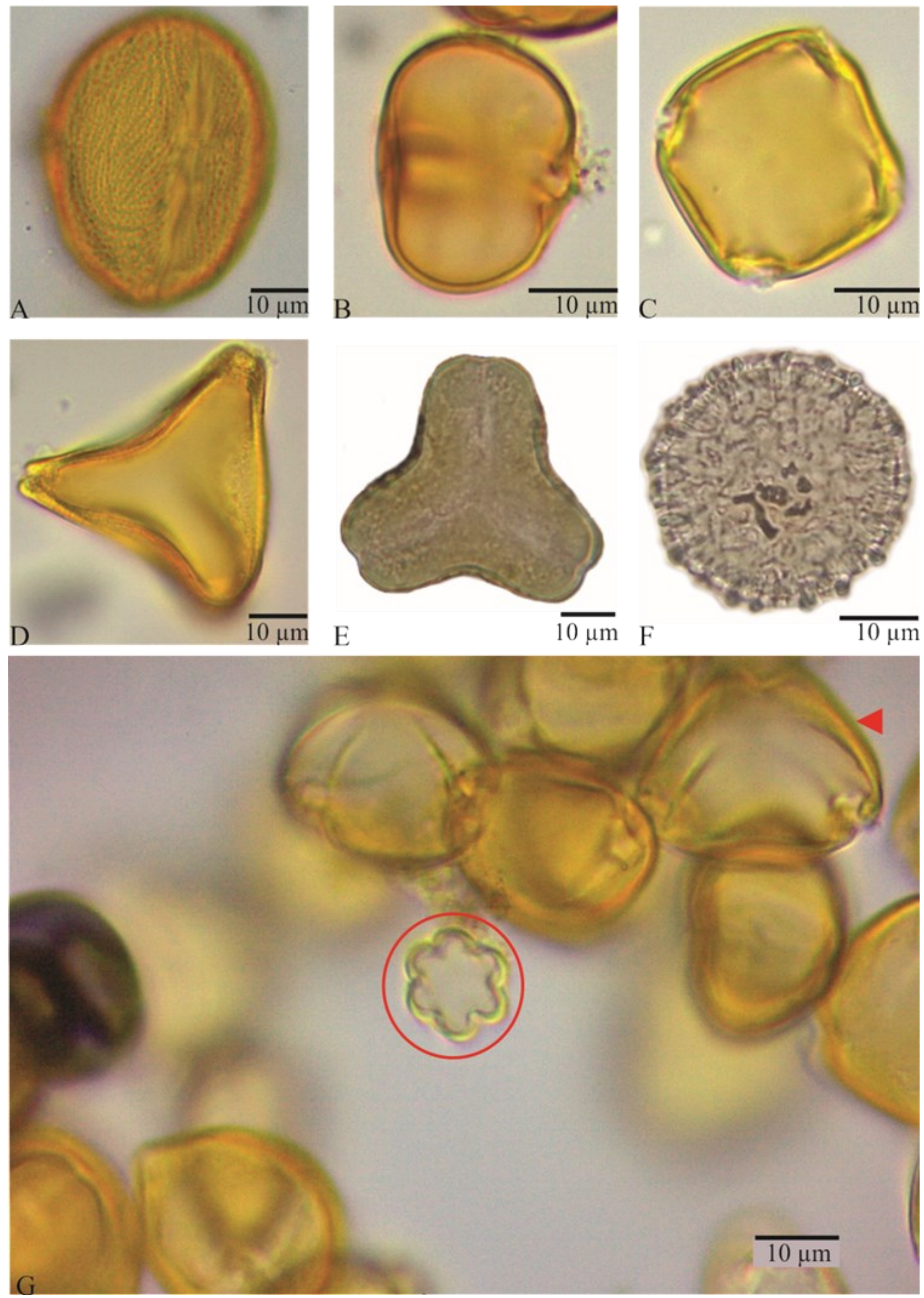
Tabela 1. Lista das espécies de abelhas estudadas em melissopalinologia ou Palinoecologia $(*)$ e das famílias, gêneros e espécies repres entadas no acervo da coleção de lâminas da Palinoteca do Laboratório de Botânica e Palinologia da UFOPA, incluindo espécies analisadas em palinotaxonomia (**) e/ou afins a tipos polínicos identificados em trabalhos aplicados, e respectiva classificação de acordo com a Lista Vermelha de Espécies Ameaçadas de Extinção da União Internacional para a Conservação da Natureza (IUCN, 2020): DD: Dados insuficientes; LC: Pouco preocupante; VU: Vulnerável - Espécies de abelhas: CCa*: Centris (Centris) aenea; CMa*: Centris (Melacentris) atriventris; Cd*: Centris (Ptilotopus) denudans; Cf*: Centris (Centris) flavifrons; Fl: Frieseomelitta longipes; Mi: Melipona (Melikerria) interrupta; Ms: Melipona (Michmelia) seminigra aff. pernigra; Sx: Scaptotrigona aff. xanthotricha; Xc*: Xylocopa (Neoxylocopa) carbonaria e Xf*: Xylocopa (Neoxylocopa) frontalis.

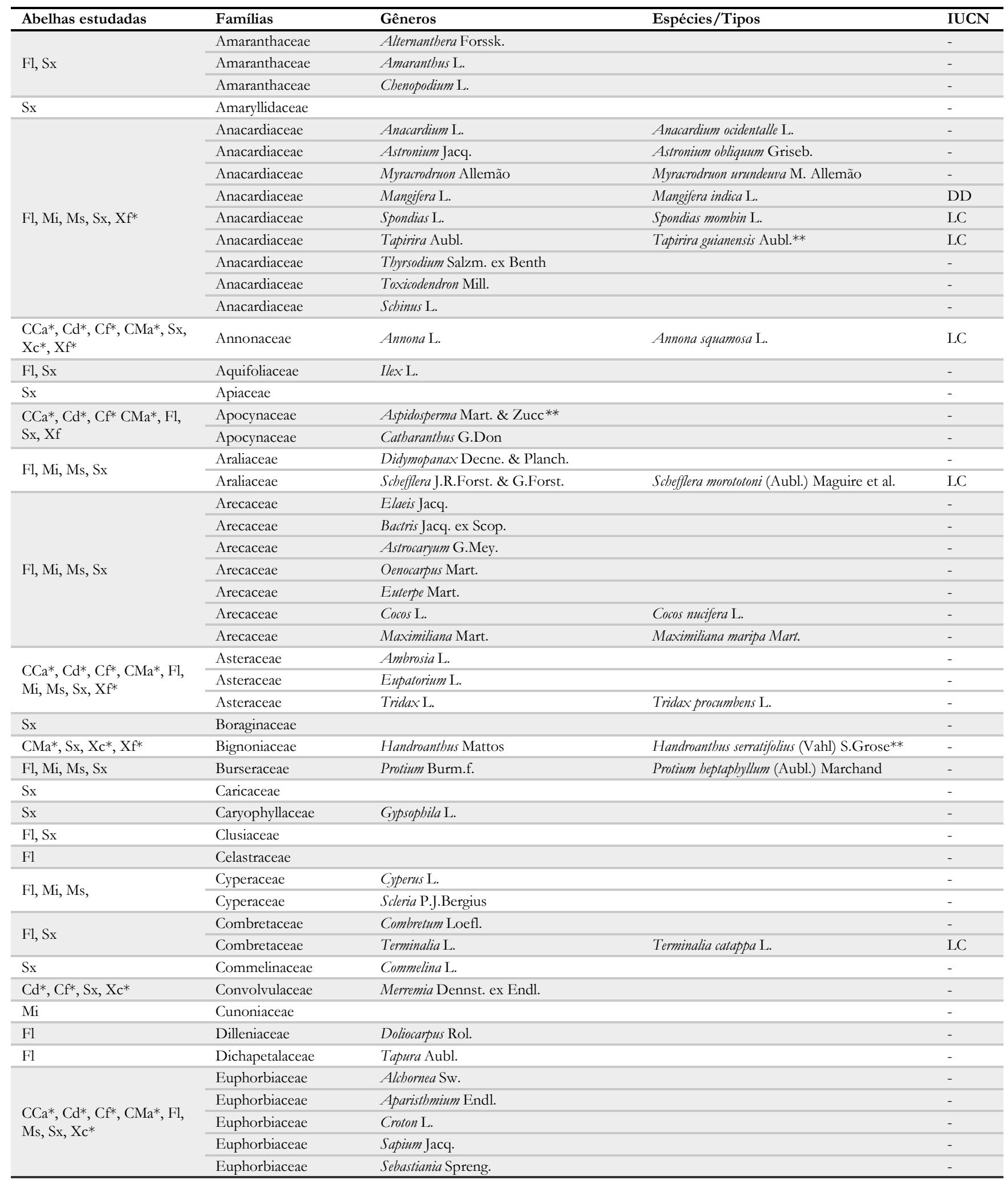


Tabela 1. Continuação.

\begin{tabular}{|c|c|c|c|c|}
\hline Abelhas estudadas & Famílias & Gêneros & Espécies & IUCN \\
\hline \multirow{45}{*}{$\begin{array}{l}\mathrm{CCa}^{*}, \mathrm{Cd}^{*}, \mathrm{Cf}^{*}, \mathrm{CMa}^{*}, \\
\mathrm{Fl}, \mathrm{Mi}, \mathrm{Ms}, \mathrm{Sx}, \mathrm{Xc}^{*}, \mathrm{Xf}^{*}\end{array}$} & Fabaceae & Aldina Endl. & & - \\
\hline & Fabaceae & Acacia Mill.** & & \\
\hline & Fabaceae & Aeschynomene $\mathrm{L}$. & Aeschynomene brasiliana (Poir.) DC & LC \\
\hline & Fabaceae & Aeschynomene & Aeschynomene marginata Benth.** & LC \\
\hline & Fabaceae & Anadenanthera Speg. & & - \\
\hline & Fabaceae & Andira Lam. & Andira parviflora Ducke** & - \\
\hline & Fabaceae & Arachis L.** & & - \\
\hline & Fabaceae & Baubinia L. & & - \\
\hline & Fabaceae & Bowdichia Kunth & Bowdichia virgilioides Kunth & $\mathrm{LC}$ \\
\hline & Fabaceae & Caesalpinia L. & Caesalpinia pluviosa DC. var. peltophoroides (Benth.) G.P.Lewis & - \\
\hline & Fabaceae & Calliandra Benth. & & - \\
\hline & Fabaceae & Cassia L. & Cassia mimosoides DC. & - \\
\hline & Fabaceae & Cenostigma Tul. & Cenostigma tocantinum Ducke & - \\
\hline & Fabaceae & Centrosema (DC.) Benth. & Centrosema brasilianum (L.) Benth.** & - \\
\hline & Fabaceae & Chamaecrista Moench & & - \\
\hline & Fabaceae & Clitoria L. & Clitoria fairchildiana R.A.Howard & LC \\
\hline & Fabaceae & Copaifera L. & Copaifera martii Hayne & LC \\
\hline & Fabaceae & Copaifera & Copaifera langsdorffii Desf. & $\mathrm{LC}$ \\
\hline & Fabaceae & Crotalaria L. & & - \\
\hline & Fabaceae & Cynometra L. & & - \\
\hline & Fabaceae & Delonix Raf. & Delonix regia (Bojer ex Hook.) Raf. & $\mathrm{LC}$ \\
\hline & Fabaceae & Desmodium Desv. & & - \\
\hline & Fabaceae & Dialium L. & & - \\
\hline & Fabaceae & Dinizia Ducke & Dinizia excelsa Ducke & LC \\
\hline & Fabaceae & Dioclea Kunth & & - \\
\hline & Fabaceae & Diplotropis Benth. & Diplotropis purpurea (Rich.) Amshoff & LC \\
\hline & Fabaceae & Hymenaea $\mathrm{L}$. & Hymenaea parvifolia Huber & $\mathrm{LC}$ \\
\hline & Fabaceae & Inga Mill. & & - \\
\hline & Fabaceae & Leucaena Benth. & Leucaena leucocephala (Lam.) de Wit** & - \\
\hline & Fabaceae & Medicago L. & Medicago sativa $\mathrm{L} . * *$ & LC \\
\hline & Fabaceae & Mimosa L. & Mimosa sensitiva $\mathrm{L}$. & - \\
\hline & Fabaceae & Mimosa & Mimosa pigra L. & - \\
\hline & Fabaceae & Mimosa & Mimosa pudica L. & LC \\
\hline & Fabaceae & Mimosa & Mimosa tenuiflora (Willd.) Poir. & LC \\
\hline & Fabaceae & Mimosa & Mimosa guilandinae (DC.) Barneby & - \\
\hline & Fabaceae & Mora Benth. & & - \\
\hline & Fabaceae & Piptadenia Benth. & & - \\
\hline & Fabaceae & Poeppigia C.Presl & Poeppigia procera C.Presl & - \\
\hline & Fabaceae & Schizolobium Vogel & Schizolobium amazonicum Huber ex Ducke & - \\
\hline & Fabaceae & Sclerolobium Vogel & Sclerolobium paniculatum Vogel & LC \\
\hline & Fabaceae & Senna Mill. & Senna polyphylla (Jacq.) H.S.Irwin \& Barneby & $\mathrm{LC}$ \\
\hline & Fabaceae & Stryphnodendron Mart. & Stryphnodendron guianense (Aubl.) Benth. & LC \\
\hline & Fabaceae & Stylosanthes Sw. & & - \\
\hline & Fabaceae & Swartzia Schreb. & Swartzia corrugata Benth.** & - \\
\hline & Fabaceae & Tamarindus L. & Tamarindus indica L.** & LC \\
\hline Sx & \multicolumn{2}{|l|}{ Flacourtiaceae } & & - \\
\hline Sx & \multicolumn{2}{|l|}{ Gentianaceae } & & - \\
\hline Sx & \multicolumn{2}{|l|}{ Gesneriaceae } & & - \\
\hline Sx & \multicolumn{2}{|l|}{ Humeriaceae } & & - \\
\hline $\mathrm{Fl}, \mathrm{Sx}$ & Lamiaceae & Hyptis Jacq. & & - \\
\hline \multirow{3}{*}{$\begin{array}{l}\mathrm{CCa}^{*}, \mathrm{Cd}^{*}, \mathrm{Cf}^{*}, \mathrm{CMa}^{*}, \\
\mathrm{Mi}, \mathrm{Ms}, \mathrm{Sx}, \mathrm{Xf}^{*}\end{array}$} & Loranthaceae & Phthirusa Mart. & & - \\
\hline & Loranthaceae & Struthanthus Mart. & & - \\
\hline & Loranthaceae & Psittacanthus Mart. & Psittacanthus eucalyptifolius (Kunth) G.Don & - \\
\hline $\mathrm{CCa}^{*}, \mathrm{Sx}, \mathrm{Ms}$ & Lythraceae & & & - \\
\hline \multirow{7}{*}{$\begin{array}{l}\mathrm{Cd}^{*}, \mathrm{Cf}^{*}, \mathrm{Fl}, \mathrm{Mi}, \mathrm{Ms}, \mathrm{Sx}, \\
\mathrm{Xc}^{*}, \mathrm{Xf}^{*}\end{array}$} & Malpighiaceae & Byrsonima Rich. ex Kunth & Byrsonima basiloba A.Juss. & LC \\
\hline & Malpighiaceae & Heteropterys Kunth & & - \\
\hline & Malpighiaceae & Malpighia L. & Malpigbia glabra L. & $\mathrm{LC}$ \\
\hline & Malpighiaceae & Lophanthera A.Juss. & Lophanthera lactescens Ducke** & - \\
\hline & Malpighiaceae & Lophanthera & Lophanthera longifolia (Kunth) Griseb.** & - \\
\hline & Malpighiaceae & Lophanthera & Lophanthera pendula Ducke** & - \\
\hline & Malpighiaceae & Lophanthera & Lophanthera spruceana Nied.** & - \\
\hline
\end{tabular}


Tabela 1. Continuação.

\begin{tabular}{|c|c|c|c|c|}
\hline Abelhas estudadas & Famílias & Gêneros & Espécies & IUCN \\
\hline \multirow{7}{*}{$\begin{array}{l}\mathrm{Cd}^{*}, \mathrm{Cf}^{*}, \mathrm{Fl}, \mathrm{Mi}, \mathrm{Ms}, \mathrm{Sx} \\
\mathrm{Xc}^{*}, \mathrm{Xf}^{*}\end{array}$} & Malvaceae & Ceiba Mill. & & - \\
\hline & Malvaceae & Eriotheca Schott \& Endl. & Eriotheca gracilipes (K.Schum.) A.Robyns & - \\
\hline & Malvaceae & Rhodognofalopsis minor A. Robyns & Rhodognofalopsis minor (Sims) A.Robyns & - \\
\hline & Malvaceae & Hibiscus L. & Hibiscus rosa-sinensis $\mathrm{L}$. & - \\
\hline & Malvaceae & Bombax L. & Bombax munguba Mart. & - \\
\hline & Malvaceae & Sida L. & & - \\
\hline & Malvaceae & Waltheria $\mathrm{L}$. & & - \\
\hline \multirow{3}{*}{$\begin{array}{l}\mathrm{Cd}^{*}, \mathrm{Cf}^{*}, \mathrm{CMa}^{*}, \mathrm{Fl}, \mathrm{Mi} \\
\mathrm{Ms}, \mathrm{Sx}, \mathrm{Xc}^{*}, \mathrm{Xf}^{*}\end{array}$} & Melastomataceae & Bellucia Raf. & & - \\
\hline & Melastomataceae & Miconia Ruiz \& Pav. & Miconia impetiolaris (Sw.) D.Don & LC \\
\hline & Melastomataceae & Mouriri Aubl. & & - \\
\hline \multirow{2}{*}{$\mathrm{Fl}, \mathrm{Sx}$} & Meliaceae & Carapa Aubl. & Carapa guianensis Aubl. & LC \\
\hline & Meliaceae & Swietenia Jacq. & Swietenia macrophylla King.** & $\mathrm{VU}$ \\
\hline $\mathrm{Fl}, \mathrm{Sx}$ & Moraceae & & & - \\
\hline \multirow{7}{*}{$\begin{array}{l}\mathrm{Cd} *, \mathrm{Cf}^{*}, \mathrm{CMa}^{*}, \mathrm{Fl}, \mathrm{Mi} \\
\mathrm{Ms}, \mathrm{Sx}, \mathrm{Xc}^{*}, \mathrm{Xf}^{*}\end{array}$} & Myrtaceae & Campomanesia Ruiz et Pav. & Campomanesia xanthocarpa (Mart.) O.Berg & - \\
\hline & Myrtaceae & Eugenia L. & Eugenia stipitata $\mathrm{McV}$ augh & LC \\
\hline & Myrtaceae & Eugenia & Eugenia brasiliensis Lam. & - \\
\hline & Myrtaceae & Myrcia DC. & Myrcia splendens (Sw.) DC. & LC \\
\hline & Myrtaceae & Psidium L. & Psidium guajava L. & LC \\
\hline & Myrtaceae & Psidium & Psidium cattleyanum Sabine & - \\
\hline & Myrtaceae & Sysygium Gaertn. & Syzygium malaccense (L.) Merr. \& L. M. Perry & LC \\
\hline $\begin{array}{l}\mathrm{Cd}^{*}, \mathrm{Cf}^{*}, \mathrm{Fl}, \mathrm{Ms}, \mathrm{Sx} \\
\mathrm{Xc}^{*}, \mathrm{Xf}^{*}\end{array}$ & Passifloraceae & Passiflora L. & & - \\
\hline $\mathrm{Fl}, \mathrm{Sx}, \mathrm{Xc} *, \mathrm{Xf}^{*}$ & Poaceae & & & - \\
\hline $\mathrm{Fl}, \mathrm{Sx}$ & Polygalaceae & & & - \\
\hline $\mathrm{Sx}$ & Portulacaceae & & & - \\
\hline \multirow{3}{*}{$\mathrm{Fl}, \mathrm{Mi}, \mathrm{Ms}, \mathrm{Sx}$} & Polygonaceae & Antigonon Endl. & & - \\
\hline & Polygonaceae & Polygonum L. & & - \\
\hline & Polygonaceae & Triplaris L. & Triplaris weigeltiana (Rchb.) Kuntze & - \\
\hline \multirow{7}{*}{$\mathrm{Fl}, \mathrm{Mi}, \mathrm{Sx}$} & Rubiaceae & Morinda L. & Morinda citrifolia $\mathrm{L}$. & - \\
\hline & Rubiaceae & $\begin{array}{l}\text { Mitracarpus Zucc. ex Schult. \& } \\
\text { Schult.f. }\end{array}$ & $\begin{array}{l}\text { Mitracarpus strigosus (Thunb.) P.L.R. Moraes, De Smedt \& } \\
\text { Hjertson }\end{array}$ & - \\
\hline & Rubiaceae & Spermacoce L. & Spermacoce capitata Ruiz \& Pav. & - \\
\hline & Rubiaceae & Borreria G.Mey. & Borreria verticillata (L.) G.Mey. & - \\
\hline & Rubiaceae & Faramea Aubl. & & - \\
\hline & Rubiaceae & Genipa L. & & - \\
\hline & Rubiaceae & Ixora L. & & - \\
\hline \multirow{2}{*}{$\mathrm{Fl}, \mathrm{Ms}, \mathrm{Sx}_{\mathrm{x}}$} & Rutaceae & Citrus L. & Citrus $\times$ limon (L.) Osbeck & - \\
\hline & Rutaceae & Zanthoxylum L. & & - \\
\hline Sx & Salicaceae & Casearia Jacq. & Casearia javitensis Kunth & - \\
\hline $\mathrm{Fl}, \mathrm{Sx}$ & Salicaceae & Casearia & Casearia guianensis (Aubl.) Urb. & - \\
\hline \multirow{5}{*}{$\mathrm{Fl}, \mathrm{Mi}, \mathrm{Ms}, \mathrm{Sx}$} & Sapindaceae & Cupania L. & & - \\
\hline & Sapindaceae & Matayba Aubl. & & - \\
\hline & Sapindaceae & Paullinia L. & & - \\
\hline & Sapindaceae & Serjania Mill. & & - \\
\hline & Sapindaceae & Talisia Aubl. & & - \\
\hline \multirow[t]{2}{*}{$\mathrm{Fl}, \mathrm{Ms}$} & Sapotaceae & Pouteria Aubl.** & & - \\
\hline & Simaroubaceae & & Simarouba amara Aubl.** & - \\
\hline \multirow{2}{*}{$\mathrm{Cd}^{*}, \mathrm{Fl}, \mathrm{Mi}, \mathrm{Ms}, \mathrm{Sx}, \mathrm{Xf}^{*}$} & Solanaceae & Acnistus Schott & & - \\
\hline & Solanaceae & Solanum L. & Solanum leucocarpon Dunal & LC \\
\hline $\mathrm{Fl}, \mathrm{Ms}$ & Urticaceae & Cecropia Loefl. & & - \\
\hline $\mathrm{Cd}^{*}, \mathrm{Cf}^{*}, \mathrm{CMa}^{*}, \mathrm{Fl}, \mathrm{Sx}$ & Verbenaceae & Lippia L. & & - \\
\hline Sx & Vitaceae & & & - \\
\hline
\end{tabular}


Tabela 2. Trabalhos desenvolvidos por estudantes de graduação e pós-graduação vinculadas(os) à palinoteca da UFOPA e ao LABOP/IBEF - UFOPA.

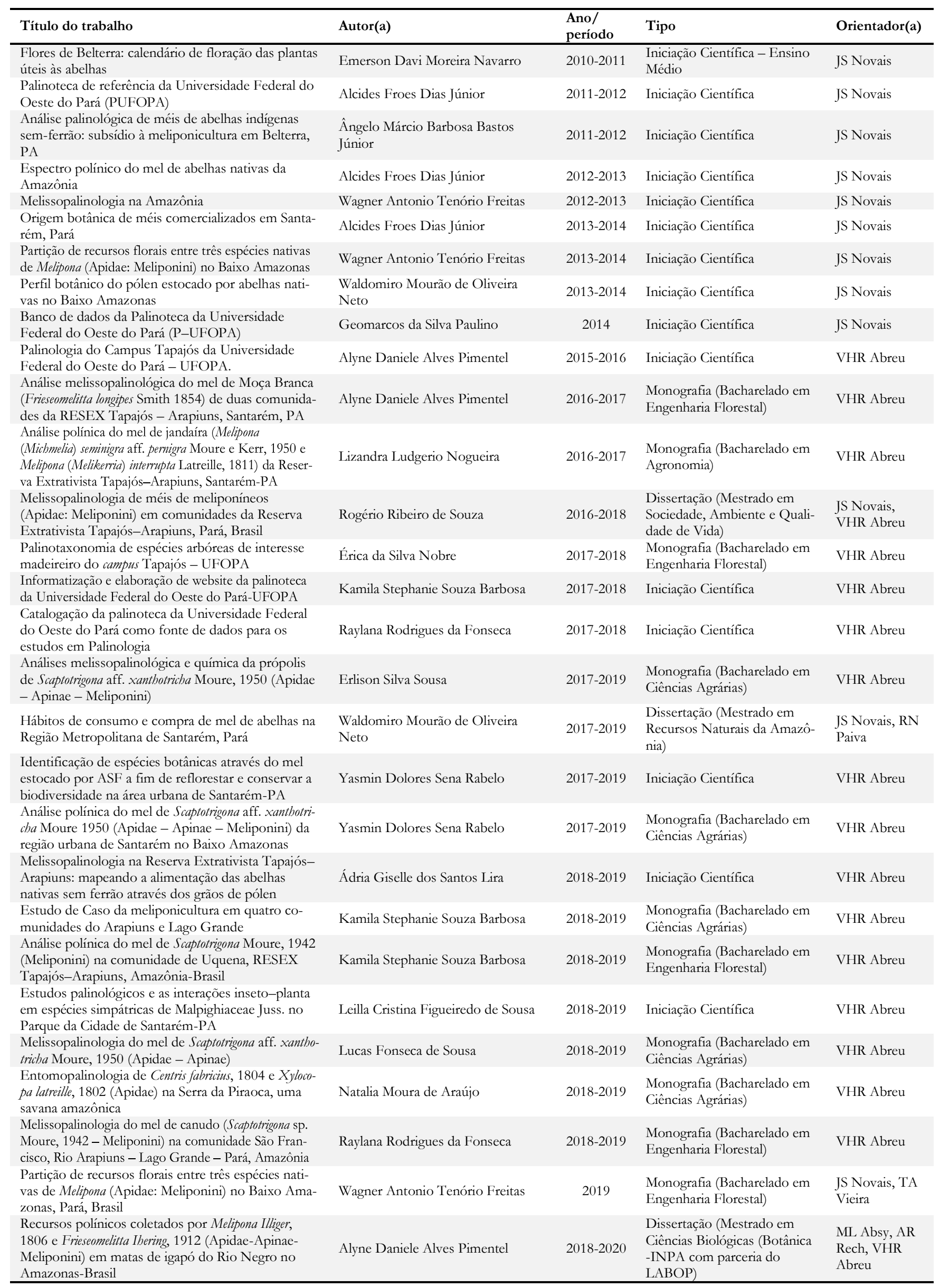




\section{Considerações Finais}

Com esse trabalho foi possível organizar a coleção de lâminas com grãos de pólen das pesquisas palinológicas na UFOPA. O acervo presente na palinoteca constitui um banco de informações que enriquece o conhecimento a respeito da diversidade polínica regional e possibilita o treinamento de recursos humanos em diferentes níveis acadêmicos nos cursos de graduação e pós-graduação da UFOPA e de outras instituições parceiras. As fotomicrografias e todas as informações palinológicas estão sendo sistematizadas e serão disponibilizadas virtualmente com livre acesso ao público representando importante avanço para o desenvolvimento científico e palinológico na região do Baixo Amazonas.

\section{Agradecimentos}

A equipe do Laboratório de Botânica e Palinologia da UFOPA agradece à FAPESPA e à PROPPIT/UFOPA, pela bolsa concedida por meio do Edital 04/2017 - PIBIC; aos laboratórios e instituições colaboradoras nas pesquisas do LABOP; aos meliponicultores e às meliponicultoras da região Oeste do Pará, pela parceria, e ao PSA Projeto Saúde e Alegria, que nos auxilia com o transporte de barco para as comunidades da RESEX Tapajós-Arapiuns.

\section{Financiamento}

Programa Institucional de Bolsas de Iniciação Científica da UFOPA Edital 04/2017 - PROPPIT/UFOPA; a Fundação Amazônia Paraense de Amparo à Pesquisa (FAPESPA) concedeu bolsa de iniciação científica à primeira autora.

\section{Contribuições de autoria}

Conceitualização: RRF, VHRA. Curadoria de dados: RRF, VHRA. Análise formal: RRF, VHRA. Aquisição de financiamento: VHRA. Investigação: RRF, VHRA. Metodologia: RRF, VHRA. Administração do projeto: VHRA. Recursos: VHRA. Programas: RRF, JSN, VHRA. Supervisão: VHRA. Validação: JSN, VHRA. Visualização: RRF, JSN, VHRA, CS. Redação - rascunho original: RRF. Redação - revisão e edição: RRF, JSN, VHRA, CS.

\section{Conflito de interesses}

Os autores declaram não haver conflitos de interesse a informar.

\section{Disponibilidade dos dados}

Os dados integrais analisados durante o estudo atual podem ser fornecidos mediante solicitação justificada à autora para correspondência.

\section{Conformidade ética}

Sempre que as pesquisas desenvolvidas pelo LABOP/UFOPA envolvem acesso a áreas protegidas, as devidas autorizações são solicitadas aos órgãos competentes.

\section{Referências}

Barth OM, Melhem TS. Glossário ilustrado de palinologia. Campinas: Unicamp; 1988.

Correia FCS, Francisco R da S, Peruquetti RC. Palinologia e a interação planta-abelha: revisão de literatura. Arquivos de Ciências Veterinárias e Zoologia da UNIPAR, Umuarama 2017;20(4):247251. doi: 10.25110/arqvet.v20i4.2017.5221

Costa JR, Morais RR, Campos LS. Cultivo e manejo do mogno (Swietenia macrophylla King). Manaus: Embrapa Amazônia Ocidental; 2013. (Documento, Embrapa Amazônia Ocidental; 114).

De Klerk P, Joosten H. The difference between pollen types and plant taxa: a plea for clarity and scientific freedom. Quaternary Science Journal 2007;56:162-171. doi: https://doi.org/10.3285/ eg.56.3.02

Erdtman G. Pollen morphology and plant taxonomy: Angiosperms. Stockholm: Almqvist \& Wiksell; 1952.

Fonseca RS, Vieira MF. Coleções botânicas com enfoque em herbário. Recurso eletrônico - 10 MB. Viçosa: UFV; 1984.

Gonçalves-Esteves V, Mendonça CBF, Santos FAR. Coleções palinológicas brasileiras. Boletín de la Asociación Latinoamericana de Paleobotánica y Palinología 2014;4:9-14.

Gonzalez M. Quantificação de custo e tempo no processo de informatização das coleções biológicas brasileiras: a experiência do herbário do Instituto de Pesquisas Jardim Botânico do Rio de Janeiro. Rodriguésia 2009;60(3):711-721. doi: http:// dx.doi.org/10.1590/2175-7860200960315

Hesse M, Halbritter H, Zetter R, Weber M, Buchner R, FroschRadivo A, Ulrich S. Pollen terminology: An illustrated handbook. New York: Springer Wien; 2009. doi: 10.1007/978-3-211-79894-2

IBAMA - Instituto Brasileiro do Meio Ambiente e dos Recursos Naturais Renováveis. Instrução normativa n. ${ }^{\circ} 160$, de 27 de abril de 2007. [Internet]. Diário Oficial [da] União. 30 abr 2007.

IUCN. A lista vermelha de espécies ameaçadas da UICN [Internet]. Versão 2020-1. [acesso em 23 mar 2020] Disponível em: https:// www.iucnredlist.org

Jones GD, Bryant JR. The use of ETOH for dilution of honey. Grana 2004;43(3):174-182. doi: 10.1080/00173130410019497

Joosten H, De Klerk P. What's in a name some thoughts on pollen classification, identification, and nomenclature in Quaternary palynology. Review of Palaeobotany and Palynology 2002;122:29 -45. doi: https://doi.org/10.1016/S0034-6667(02)00090-8

Luz, CFP, Esteves, LM, Corrêa, MAS, Cruz-Barros, MAV. A palinoteca do Núcleo de Pesquisa em Palinologia, Centro de Pesquisa em Plantas Vasculares, Instituto de Botânica, São Paulo, Brasil. Boletín de la Asociación Latinoamericana de Paleobotánica y Palinología 2014;14:155-161.

Marques AC, Lamas CJE. Taxonomia zoológica no Brasil: estado da arte, expectativas e sugestões de ações futuras. Papéis Avulsos de Zoologia 2006;46(13):139-174. doi: 10.1590/S003110492006001300001

Marques-Souza AC, Miranda IPA, Moura CO, Rabelo A, Barbosa EM. Características morfológicas e bioquímicas do pólen coletado por cinco espécies de meliponíneos da Amazônia Central. Acta Amazonica 2002;32:217-229. doi: 10.1590/1809$\underline{43922002322229}$

Melhem TS, Barros MAVC, Corrêa AMS, Makino-Watanabe H, Silvestre-Capelato MSF, Gonçalves-Esteves V. Variabilidade polínica em plantas de Campos do Jordão (São Paulo, Brasil). Boletim do Instituto de Botânica 2003;16:16-104.

Novais JN, Absy ML, Santos FAR. Pollen grains in honeys produced by Tetragonisca angustula (Latreille, 1811) (Hymenoptera: Apidae) in tropical semi-arid areas of north-eastern Brazil. Arthropod-Plant Interactions 2013;7:619-632. doi: 10.1007/s11829-013-9276-x

Novais JS, Araújo VLR, Silveira AS, Bandeira MSF. palinoFLORAS: uma palinoteca vinculada a um jardim botânico no sul da 
Bahia, Brasil. Paubrasilia 2018;1(1): 20-24. doi: $10.33447 /$ paubrasilia.v1i1.2

Oliveira NTB, Lorscheitter ML. Palinologia de espécies da ordem Apiales da flora do Rio Grande do Sul. Porto Alegre: UFRGS; 2014.

Punt W, Hoen PP, Blackmore S, Nilsson S, Le Thomas A. Glossary of pollen and spore terminology. Review of Palaeobotany and Palynology 2007;143(1-2):1-81. doi: $10.1016 /$ j.revpalbo.2006.06.008

Salgado-Labouriau ML, Vanzolini PE, Melhem TS. Variation of polar axes and equatorial diameters in pollen grains of two species of Cassia. Grana Palynologica 1965;6:166-176. doi: $\underline{10.1080 / 00173136509429142}$

Salgado-Labouriau ML. Contribuição à palinologia dos cerrados. Rio de Janeiro: Academia Brasileira de Ciências; 1973.

Silva LAE, Fraga CN, Almeida TMH, Gonzalez M, Lima RO, Rocha MS, Bellon E, Ribeiro RS, Oliveira FA, Clemente LS, Magdalena UR, Medeiros EVS, Forzza RC. Jabot-Sistema de Gerenciamento de coleções botânicas: a experiência de uma década de desenvolvimento e avanços. Rodriguésia 2017;68(2):391-410. doi: $\underline{10.1590 / 2175-7860201768208}$

Villas-Boas J. Manual tecnológico: mel de abelhas sem ferrão. Brasília: Instituto Sociedade, População e Natureza; 2012.

\section{Catalogando la palinoteca de la Universidad Federal del Oeste de Pará como fuente de da- tos para estudios en palinología}

Describimos el catálogo de las láminas palinológicas de la palinoteca de la Universidad Federal del Oeste de Pará. Actualmente, la palinoteca tiene 863 láminas en su colección. Dado que el trabajo en palinología ha sido intenso en esta universidad desde 2010, existen más de 1,000 láminas para incorporar a la colección. La palinoteca ha apoyado actividades de investigación y enseñanza de palinología, enriqueciendo el conocimiento de la diversidad regional de polen, permitiendo la capacitación de estudiantes de pregrado y posgrado que trabajan en el área. Toda la información palinológica está siendo sistematizada y estará disponible a través de un sitio web de libre acceso al público, con el propósito de promover la divulgación científica de la palinología, lo que representa un avance importante y contribuirá al desarrollo de la palinología en la región amazónica.

Palabras clave: Granos de polen. Melisopalinología. Colección biológica.

Catalogage de la palinothèque de l'Université Fédérale du Pará Occidental comme source de données pour les études en palynologie

Nous décrivons le catalogage des diapositives palynologiques de la palynothèque de l'Université Fédérale du Pará Occidental. Actuellement, la palinoteca compte 863 diapositives dans sa collection. Comme le travail en palynologie est intense dans cette université depuis 2010, il y a plus de 1000 diapositives à incorporer dans la collection. La palynothèque a subventionné des activités de recherche et d'enseignement de la palynologie, enrichissant les connaissances sur la diversité des pollens régionaux, permettant la formation d'étudiants de premier cycle et des cycles supérieurs qui travaillent dans la région. Toutes les informations palynologiques sont systématisées et seront mises à disposition via un site avec un accès gratuit au public, dans le but de promouvoir la diffusion scientifique de la palynologe. Cela représente une avancée importante et contribue au développement de la palynologie dans la région amazonienne.

Mots clés: Grains de pollen. Mélissopalinologie. Collection biologique. 